\title{
Back-streaming ion emission and beam focusing on high power linear induction accelerator
}

\author{
Jun Zhu, Nan Chen, Haijun Yu, Xiaoguo Jiang, Yuan Wang, Wenhua Dai, \\ Feng Gao, Minhong Wang, Jin Li, and Jinshui Shi \\ Institute of Fluid Physics, CAEP, Mianyang, Sichuan, 621900, China
}

(Received 12 January 2011; published 3 August 2011)

\begin{abstract}
Ions released from target surfaces by impact of a high intensity and current electron beam can be accelerated and trapped in the beam potential, and further destroy the beam focus. By solving the 2D Poisson equation, we found that the charge neutralization factor of the ions to the beam under space charge limited condition is $1 / 3$, which is large enough to disrupt the spot size. Therefore, the ion emission at the target in a single-pulse beam/target system must be source limited. Experimental results on the time-resolved beam profile measurement have also proven that. A new focus scheme is proposed in this paper to focus the beam to a small spot size with the existence of back-streaming ions. We found that the focal spot will move upstream as the charge neutralization factor increases. By comparing the theoretical and experimental focal length of the Dragon-I accelerator ( $20 \mathrm{MeV}, 2.5 \mathrm{kA}, 60 \mathrm{~ns}$ flattop), we found that the average neutralization factor is about $5 \%$ in the beam/target system.
\end{abstract}

DOI: 10.1103/PhysRevSTAB.14.082801

\section{INTRODUCTION}

High resolution $\mathrm{x}$-ray radiography requires the production of a small spot on the surface of a bremsstrahlung converter target by a pulsed high-current relativistic electron beam [1]. However, positive ions pulled out from impurities at the target surface or the preexisting thermal plasma by the electron beam's space charge force can affect the beam focus. As the ions propagate upstream, they will partially charge neutralize the electron beam, resulting in radial pinching and subsequent diverging of the beam. Therefore, the final beam spot size on the target will be larger than expected. Protons have greater potential to destroy the beam focus since they travel upstream at a much higher speed and form a longer ion channel.

In recent years, there have been many experimental, theoretical, and simulation studies to understand the influence of the ions to the beam spot size. In the theoretical model, the ion emission is assumed to be space charge limited and follow the planar Child-Langmuir law. The ion channel formed in $40 \mathrm{~ns}$ was predicted to be long enough to disrupt the beam spot in an idealized system [2]. The experiments on the DARHT-I linear induction accelerator (LIA) have also proven the existence of ions [3]. A streak camera was used to record the Cherenkov radiation coming from a vertical oriented quartz fiber impacted by the incident electron beam. Different metal foils were placed upstream of the quartz fiber to generate ions when interacting with the beam. In these experiments, divergence of the electron beam was observed and the ions are believed to

Published by the American Physical Society under the terms of the Creative Commons Attribution 3.0 License. Further distribution of this work must maintain attribution to the author(s) and the published article's title, journal citation, and DOI.
PACS numbers: 41.75.Ht, 41.85.Ew, 41.85.Ja, 41.85.Lc

come from thermal desorption and subsequent impact ionization. However, the Dragon-I (20 MeV, $2.5 \mathrm{kA}, 70 \mathrm{ns)} \mathrm{[4]}$ and DARHT-I (20 MeV, $1.8 \mathrm{kA}, 60 \mathrm{~ns})$ [5] accelerators have both achieved a 50\% modulation transfer function spot size smaller than $2.0 \mathrm{~mm}$ and there is no special design to restrict the ions. On the other hand, experimental observation on the ETA-II accelerator confirmed the beam disruption in the presence of a target plasma initiated by another electron beam [6]. It is apparent that a complete understanding of the physics of the ion emission and the growth of the beam spot size is still to come.

We have investigated the ion emission and beam spot size variation both experimentally and theoretically. In Sec. II, we will introduce our theoretical model of the ion emission from a target surface. In Sec. III, we will present how to focus the electron beam in a single-pulse LIA with the back-streaming ions, and explain why the spot size will disrupt with the target plasma. Section IV shows our experimental results on the Dragon-I LIA. The influences of the ions to the beam were observed, and we proved that the ion emission in a single-pulse beam/target system is source limited. A discussion is presented in Sec. V.

\section{SPACE CHARGE LIMITED EMISSION OF IONS}

The stable propagation of a relativistic electron beam in vacuum requires balance between the electric and magnetic forces. For a charge unneutralized relativistic electron beam, the difference between its self-electric force and magnetic force scales as $1 / \gamma^{2}$, where $\gamma$ is the relativistic factor. The electric force always dominates slightly so the beam will diverge as it propagates in vacuum. However, if the charge of the electron beam is neutralized by ions, the dynamics of propagation can be quite different. The net force acting on the beam will scale $\left(f-1 / \gamma^{2}\right)$, where $f$ is 
the charge neutralization factor. For a highly relativistic electron beam, a small fractional charge neutralization can cause the magnetic force to dominate, resulting in radial pinching and subsequent diverging after the pinch point. Therefore, $f$ is a key parameter in studying the beam focus with the back-streaming ions. We will use a twodimensional axisymmetric model, as shown in Fig. 1, to find out the value of $f$ when the ion emission is space charge limited.

The two-dimensional Poisson equation can be written as

$$
\frac{1}{r} \frac{\partial}{\partial r}\left(r \frac{\partial \Phi}{\partial r}\right)+\frac{\partial^{2} \Phi}{\partial z^{2}}=\frac{-j_{i}(r)}{\varepsilon_{0}\left(-2 q \Phi / m_{i}\right)^{1 / 2}}+\frac{\rho_{e}(r)}{\varepsilon_{0}} .
$$

Let us assume that the beam potential is separable in $r$ and $z$ :

$$
\Phi(r, z)=-\phi(r) \psi(z) .
$$

Substitution of Eq. (2) into Eq. (1) yields

$$
\varphi(r) \psi(z)+\phi(r) \psi^{\prime \prime}(z)=\frac{j_{i}}{\varepsilon_{0}\left(2 q \phi(r) \psi(z) / m_{i}\right)^{1 / 2}}-\frac{\rho_{e}}{\varepsilon_{0}},
$$

where

$$
\varphi(r)=\frac{1}{r} \frac{\partial}{r}\left(r \frac{\partial \phi}{\partial r}\right)
$$

If we multiply Eq. (3) by $2 d \psi / d z$ and integrate on both sides, and further use the boundary condition $\psi(0)=0$, we can obtain

$$
\psi^{\prime}(z)^{2}=\mathrm{A} \psi^{2}(z)-B \psi(z)+C \sqrt{\psi(z)}+D,
$$

where $D$ is the constant of integration and the coefficients $A, B, C$ are in the form of

$$
\begin{gathered}
A=-\frac{\varphi(r)}{\phi(r)} \\
B=\frac{2 \rho_{e}}{\varepsilon_{0} \phi(r)} \\
C=\frac{4 j_{i}}{\phi(r)^{3 / 2} \varepsilon_{0}(2 q / m)^{1 / 2}} .
\end{gathered}
$$

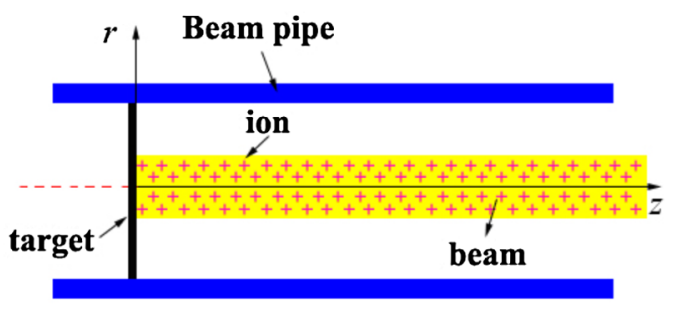

FIG. 1. Geometry model.
It is obvious that the right side of Eq. (5) cannot be negative, and for $z \rightarrow \infty$ we expect $d \psi / d z=0$. That is to say, the minimum of the right side of Eq. (5) is zero. Define

$$
y=\sqrt{\psi}
$$

and

$$
\delta=A y^{4}-B y^{2}+C y+D .
$$

We will have

$$
\left.\frac{d \delta}{d y}\right|_{z \rightarrow \infty}=4 A \Lambda^{3}-2 B \Lambda+C=0
$$

and

$$
A \Lambda^{4}-B \Lambda^{2}+C \Lambda+D=0,
$$

where

$$
\Lambda=\sqrt{\psi_{z \rightarrow \infty}}
$$

The charge neutralization fraction of the beam by the ions can be written as

$$
f=\frac{j_{i} / \sqrt{2 q \phi \psi_{z \rightarrow \infty} / m}}{\rho_{e}} .
$$

Substituting Eqs. (7) and (8) into (14), we can find that

$$
C=2 f B \Lambda \text {. }
$$

Further substituting Eq. (15) into (11) and (12), we will get

$$
\begin{gathered}
A=(1-f) B / 2 \Lambda^{2} \\
D=(1-3 f) B \Lambda^{2} / 2 .
\end{gathered}
$$

Using the boundary condition $\psi^{\prime}(0)=0$ for the space charge limited emission, we can determine that $D$ must be zero. So we can immediately find that the charge neutralization factor $f$ must be $1 / 3$ in the space charge limited condition. Note that $f$ is a constant and independent of the beam parameters, the beam profile distribution, the radius of the beam pipe, and the type of the ion.

Substituting Eqs. (9) and (15)-(17) into (5) we will have

$\frac{2 y d y}{d z}=\sqrt{B\left(\frac{1-f}{2 \Lambda^{2}} y^{4}-y^{2}+2 f \Lambda y+\frac{(1-3 f) \Lambda^{2}}{2}\right)}$.

To find the potential drop in the vicinity of the target, Eq. (18) can be written in the form

$$
\frac{\Lambda}{\sqrt{B}} \int \frac{p}{\sqrt{(1-f) p^{4} / 2-p^{2}+2 f p+(1-3 f) / 2}} d p=\frac{z}{2},
$$

where $p=y / \Lambda$. Note that $p$ is independent of $r$, so that Eq. (19) will have a solution only when $\Lambda / B^{1 / 2}$ is constant.

For $\Lambda / B^{1 / 2}=$ const, we will find that the potential and electron density distribution are in the form of

$$
\phi(r)=J_{0}\left(x_{01} r / R\right)
$$


and

$$
\rho_{e}=\rho_{0} J_{0}\left(x_{01} r / R\right),
$$

where $J_{0}$ is the zeroth order Bessel function, $x_{01}$ is the first root of $J_{0}$, and $\rho_{0}$ is the on-axis charge density of the beam. The distribution function given by Eqs. (20) and (21) represents a special case that the radius of the beam pipe and beam are equal. Since $\rho_{0}$ can be obtained by integrating over the beam profile

$$
\int_{0}^{R} 2 \pi r \rho_{0} J_{0}\left(x_{01} r / R\right) d r=\frac{I}{\beta c}
$$

we find that

$$
\rho_{0}=\frac{x_{01} I}{2 \pi \beta c R^{2} J_{1}\left(x_{01}\right)} .
$$

Substituting Eqs. (20), (21), and (23) into (6), (7), and (16), we will have

$$
\begin{aligned}
& \Lambda / \sqrt{B}=R \sqrt{(1-f) / 2} / x_{01} \\
& \Lambda=R \sqrt{(1-f) \rho_{0} / \varepsilon_{0}} / x_{01} .
\end{aligned}
$$

Now, we can integrate the left side of Eq. (19) numerically for different parameters. The distributions of the space charge induced potential near the target for different $f$ are plotted in Fig. 2. Here we have used the parameters of $I=2.5 \mathrm{kA}$ and $R=1.5 \mathrm{~mm}$. As $f$ increases, the maximum potential changes from $-120 \mathrm{kV}$ to $-80 \mathrm{kV}$ due to the increasing charge neutralization effect. The potential distributions in the vicinity of the target for different beam radius are plotted in Fig. 3. The results indicate that the potential changes rapidly over a distance of about 3 times the beam radius. For ions moving at a velocity of several $\mathrm{cm} / \mathrm{ns}$, the ion channel formed in $60 \mathrm{~ns}$ will be dozens of centimeters long. So we will assume that the charge

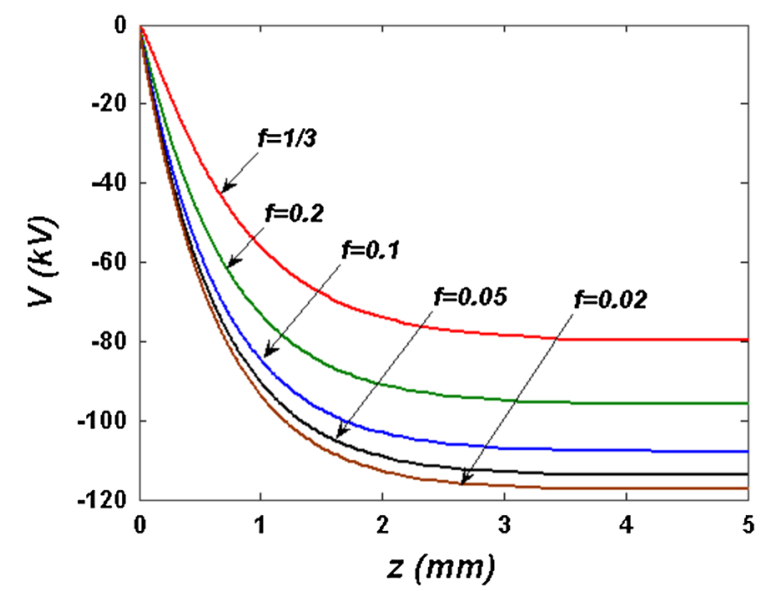

FIG. 2. Potential distribution in the vicinity of the target for different charge neutralization factor $f$.

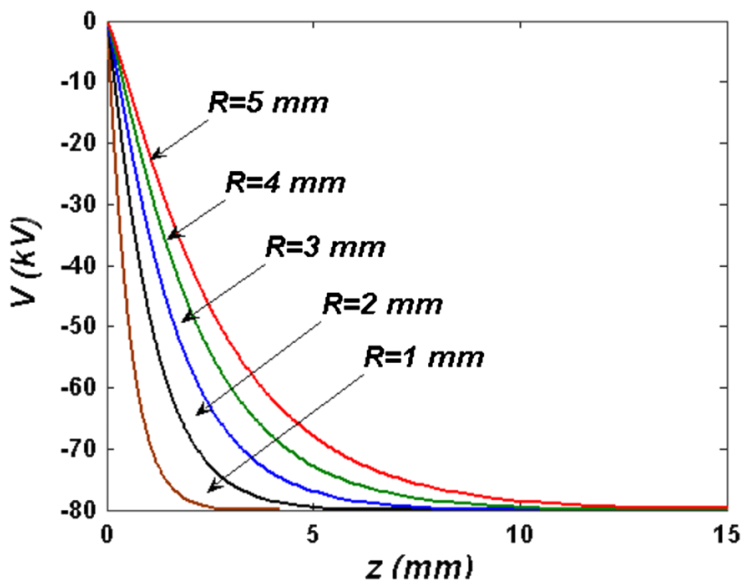

FIG. 3. Potential distribution in the vicinity of the target for different beam radius $R$.

neutralization factor is constant in the entire ion channel in the following calculation.

\section{BEAM FOCUSING WITH ION EMISSION}

The influence of such an ion channel on the beam focus can be estimated by the envelope equation [7],

$$
R^{\prime \prime}=-\left[\frac{e B_{z}}{2 \beta \gamma m_{0} c}\right]^{2} R+\frac{2 I}{\gamma \beta^{3} I_{0} R}\left(\frac{1}{\gamma^{2}}-f\right)+\frac{\varepsilon^{2}}{R^{3}},
$$

where $R=R(z)$ is the beam's edge radius, $B_{z}$ is the final focus magnetic field, $I, \gamma$, and $\varepsilon$ are the beam current, the relativistic factor, and the unnormalized beam's edge emittance, respectively, $I_{0}$ is the Alfven current, and $f$ is the charge neutralization factor.

The time-varied beam envelops with different $f$ are calculated by numerically solving Eq. (26). The result for $f=1 / 3$ is plotted in Fig. 4. It indicates that the spot size at the target will first reduce slightly and then increase

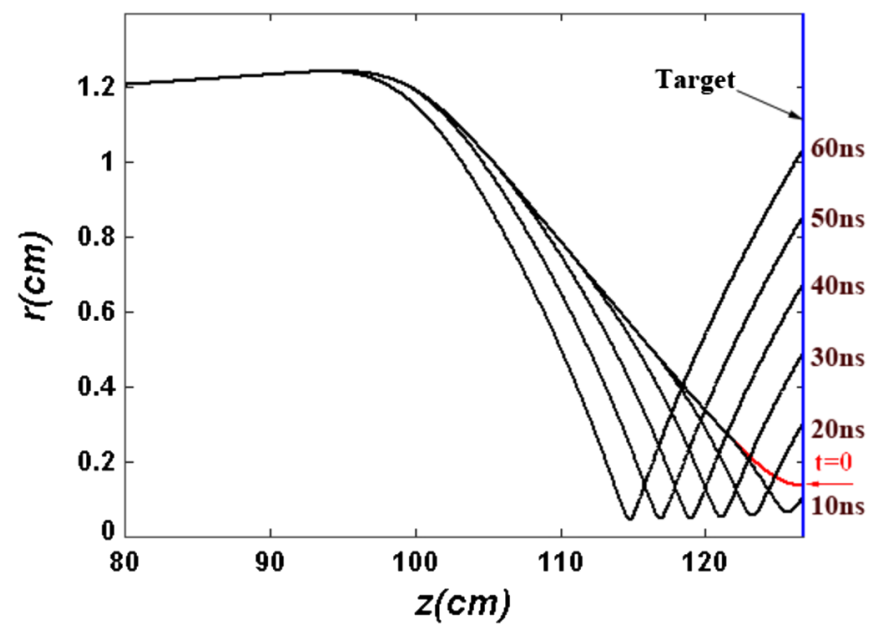

FIG. 4. Time-dependent beam envelop for space charge limited ion emission $(f=1 / 3)$. 
rapidly. Therefore, the beam spot size at the target will disrupt with a preformed plasma on the target surface. The time-varied spot sizes for different $f$ are plotted in Fig. 5. It is obvious that the spot size at the end of the pulse will be at least twice as large as the initial one when $f$ is greater than 0.05 . For $f=0.02$, the spot size will nearly maintain the same during the pulse time. However, the experimental results on the DARHT-I LIA have proven that the neutralization factor $f$ is large enough to cause the radius of the beam to change rapidly.

In order to focus the beam with ions released from the target surface, we proposed a new focus scheme. The target will be moved upstream to achieve a relative large spot size at the beginning of the pulse. When the ions propagate upstream, the spot size at the target will first reduce due to the ions' charge neutralization effect and then increase. The simulated result for $f=0.05$ is shown in Fig. 6, and the variations of the spot size at the target for different $f$ are shown in Fig. 7. The comparison between the average spot sizes at the target for both focusing schemes is shown in Fig. 8. It is apparent that the average spot size reduces

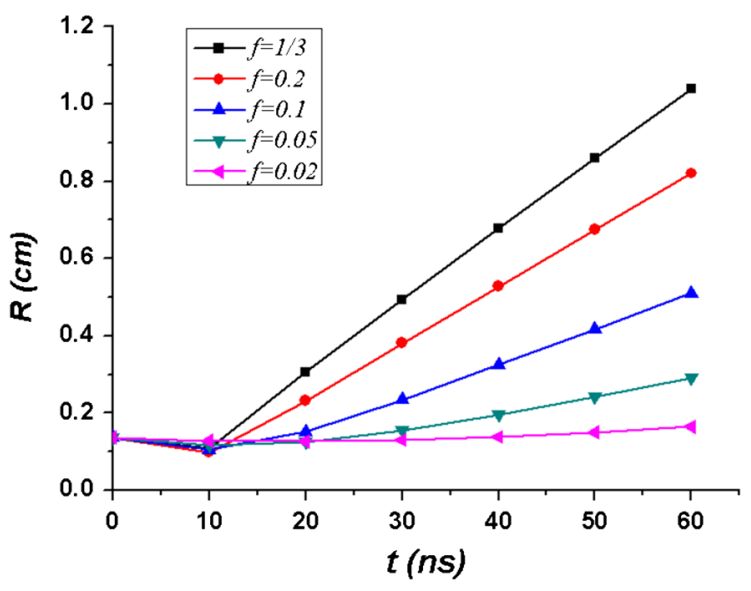

FIG. 5. Spot size variation for different $f$.

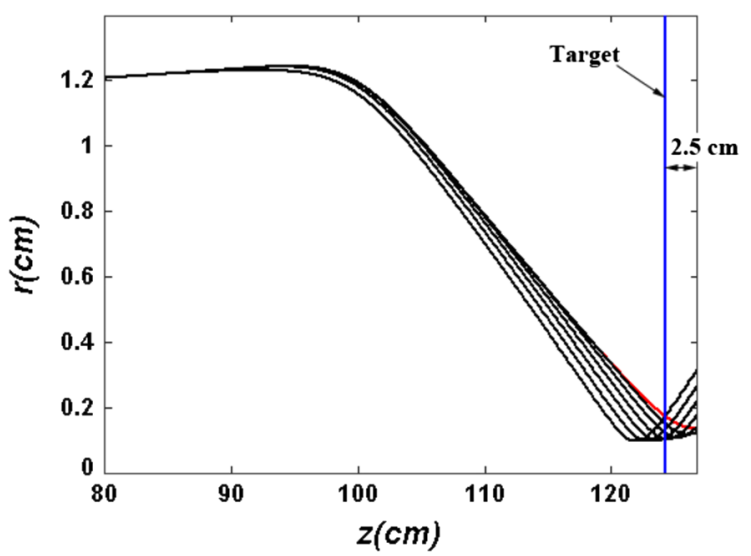

FIG. 6. Time-dependent beam envelops for $f=0.05$ using a new focusing scheme.

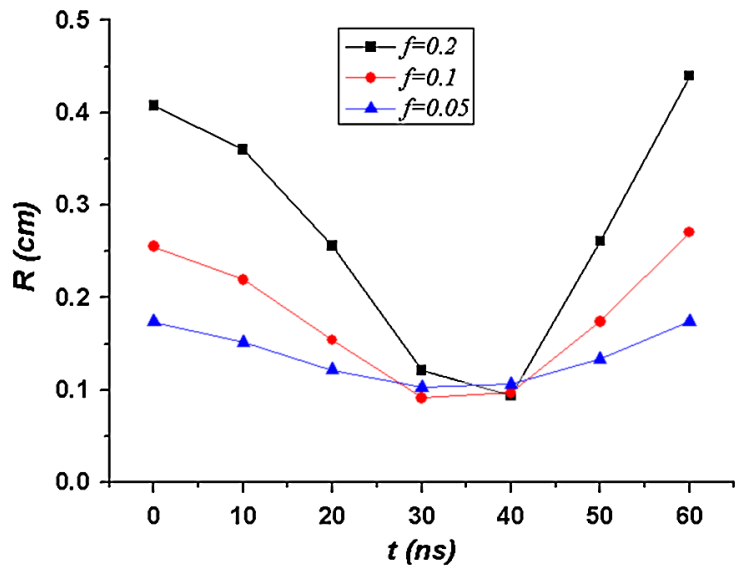

FIG. 7. Spot size variation for different $f$ using a new focusing scheme.

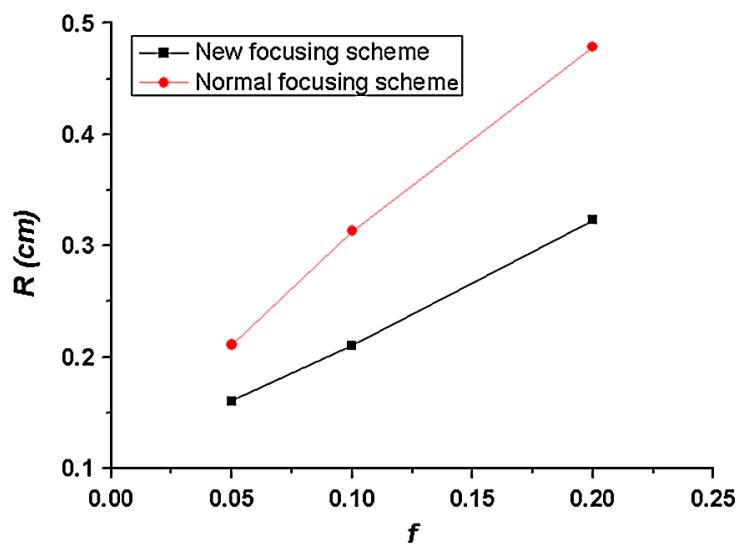

FIG. 8. Comparison between the average spot sizes at the target for both focusing schemes.

considerably when the new focusing scheme is used. Moreover, the focal spot will move upstream continuously as $f$ increases.

Actually, the calculated focal length for the Dragon-I LIA is $25.9 \mathrm{~cm}$ by using both the envelop equation and a nonlaminar and noncollision numerical model [8], while the experimental focal length is only $23.2 \mathrm{~cm}$. So the focal spot moves $2.7 \mathrm{~cm}$ upstream comparing with the simulated result. Therefore, we can infer that the charge neutralization factor in the Dragon-I beam/target system is a little bit larger than 0.05 .

\section{EXPERIMENTS ON THE DRAGON-I LIA}

A time-resolved measurement system for the electron beam's profile [9] was used to observe the influence of the ions on the beam propagation. The optical transition radiation (OTR) foil produces OTR while electrons are impacting on the foil. A high speed multiframe CCD camera (3 ns exposure time, $10 \mathrm{~ns}$ frame interval) was used to record the light coming from the front surface of the OTR foil. The experimental setup is shown in Fig. 9. In order to generate 
ions, a thin target foil was installed at the upstream of the OTR foil. The foil thickness is minimized to reduce the beam scattering. The influence of ions on the beam propagation can be obtained by comparing the experimental results with different target foils or without any target foil.

The measured beam profile at the OTR foil is quite different from the spot size at the focal spot since the beam was focused at the target foil and drifted freely for a distance of about $36 \mathrm{~cm}$ to the OTR foil. Moreover, ions can propagate on each side of the target foil in this system, rather than just on the front surface when the beam is interacting with a thick bremsstrahlung target, which will enhance the effect.

The typical time-resolved beam profile without any target foil is shown in Fig. 10(a). The radius of the beam spot increases slightly during the first $30 \mathrm{~ns}$ and then becomes constant. The hollow beam is obscure and has not been observed in our previous experiments [9], in which the beam profile was measured after the last induction cell and before the final focusing solenoid. The experimental result with a molybdenum foil $(10 \mu \mathrm{m})$ is shown in Fig. 10(b). The small bright spot in the center of each image is due to the radiation produced by the beam and target foil interaction. Since the surface of the OTR foil is a mirror surface, the image of the radiation area can be recorded by the camera through the OTR foil and the reflection mirror. Comparison between the radius of the beam spot for the above two cases is shown in Fig. 11. With the existence of a molybdenum foil, the radius of the beam spot increases faster in the first $40 \mathrm{~ns}$ and then decreases.

In order to validate if the ion emission during singlepulse beam/target interaction is space charge limited or not, a Kapton film $(50 \mu \mathrm{m})$ was used as the target foil. When an electron beam impacts on a dielectric film, the potential drop along the beam profile $(75 \mathrm{kV}$ for the Dragon-I beam) will be large enough to cause flashover along the surface. The physics of the surface flashover has been studied intensively in the past, and the final stage of the flashover in such a case was believed to be discharge through the desorbed gas along the dielectric surface [10]. During the discharge, a large amount of plasma will be

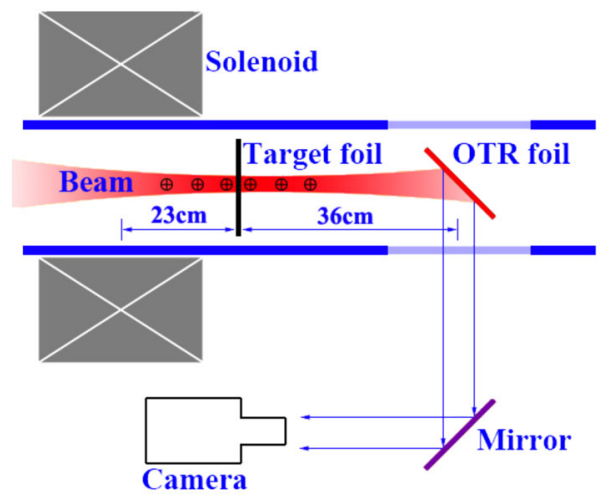

FIG. 9. Experimental setup.
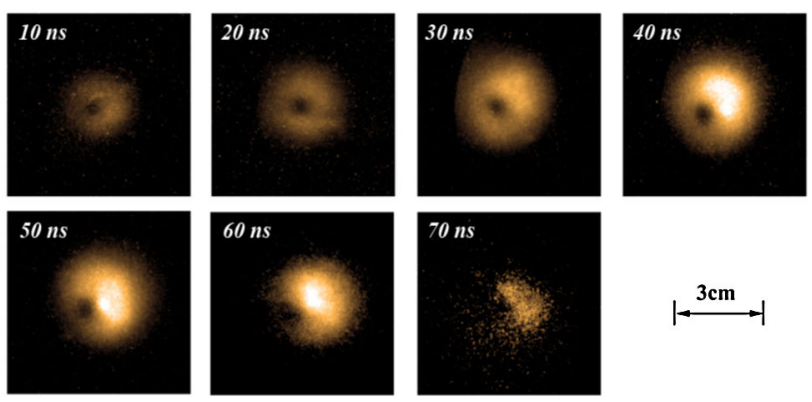

(a)
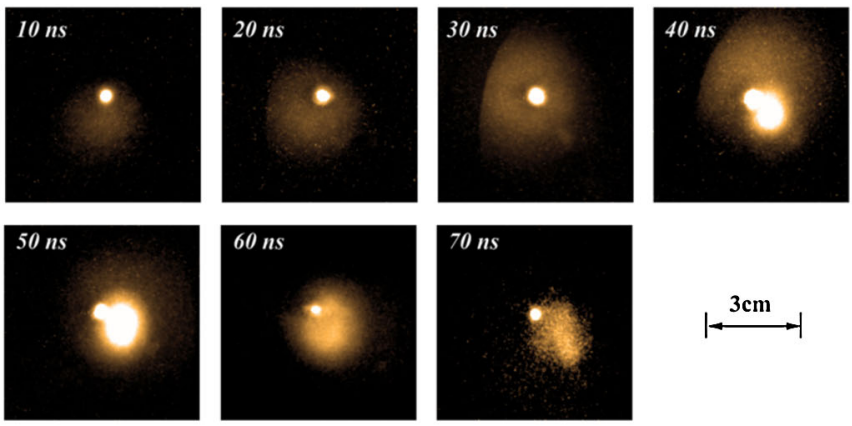

(b)
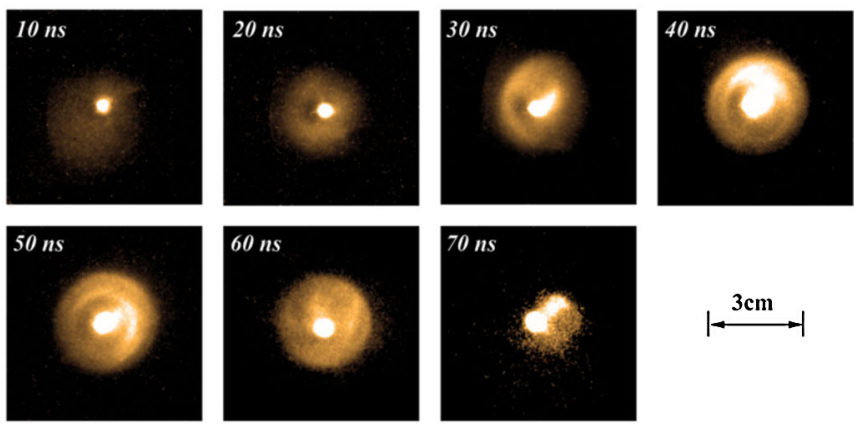

(c)
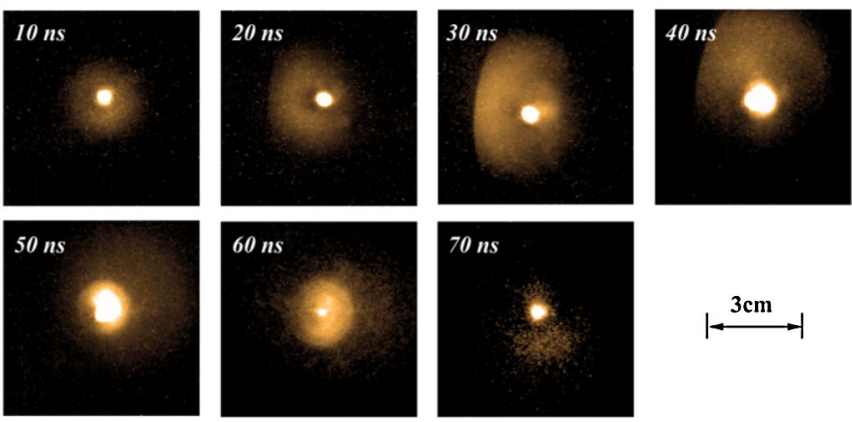

(d)

FIG. 10. Sequence of beam profiles with (a) none; (b) $10 \mu \mathrm{m}$ Mo; (c) $50 \mu \mathrm{m}$ Kapton; and (d) $50 \mu \mathrm{m}$ aluminized Kapton.

produced, which will provide a copious source of positive ions. Therefore, the ion current from the Kapton surface should be larger than that from the molybdenum surface, although whether it is space charge limited or not is still unknown. The typical experimental result with a Kapton 


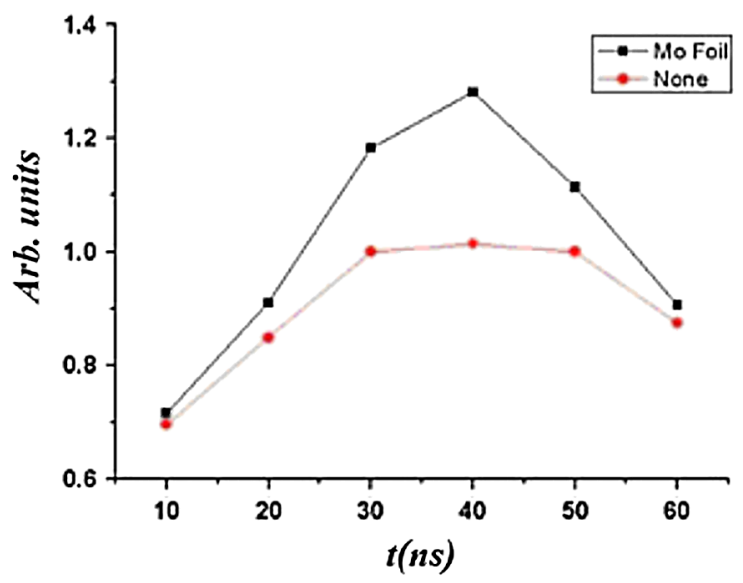

FIG. 11. Comparison between the radius of the beam spot variation with and without a target foil.

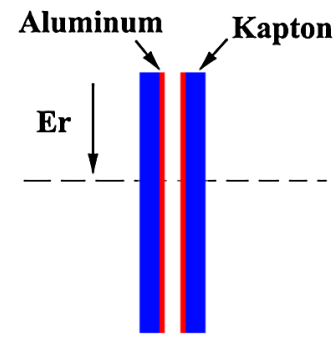

FIG. 12. Schematic of the aluminized Kapton foil.

film is shown in Fig. 10(c). The variation of the beam profile is totally different from the result given in Fig. 10(b). The radius of the beam spot does not change rapidly. However, some distinct transverse motion was observed inside the beam.

In order to further confirm that the surface flashover will increase the ion current, an aluminized Kapton foil was used, as shown in Fig. 12. We fold the 25- $\mu$ m-thick aluminized Kapton foil to make the surface of the Kapton outside and the surface of the aluminum inside. When the beam impacted on the target foil, the aluminum plate inhibited the surface flashover while the ions still emitted from the Kapton surface due to thermal desorption. The experimental result with such a foil is shown in Fig. 10(d). During the first $40 \mathrm{~ns}$, the variation of the beam profile is similar with the result in Fig. 10(b). After that, the aluminum plate became "disabled" due to hydrodynamic expansion, thus surface flashover occurred along the Kapton surface. Since the surface flashover increased the ion emission from the surface of the target foil, the following development of the beam profile is totally different from the result in Fig. 10(b).

\section{DISCUSSION}

The experiments and analysis presented strongly suggest that the ion emission in a single-pulse electron beam/target system is not space charge limited. A new focusing scheme is proposed in this paper to focus the beam to a small spot size with the existence of the ions. By using this focusing scheme, the focal length will reduce comparing to the theoretical one.

Because the components of the surface contaminants and the cross section for electron impact ionization at $20 \mathrm{MeV}$ are both unknown, we assume that protons are the only ion in our numerical simulation. The impact of this assumption on the simulation result needs further study, although Vermare [3] found that protons must be a significant component of the ions. Since the beam spot will be totally disrupted with a preformed plasma on the target surface, research on how to focus the electron beam pulse in such a case is our ongoing priority.

[1] J. J. Deng et al., in Proceedings of the 21st International Linac Conference, Gyeongju, Korea, 2002 (Pohang Accelerator Laboratory, Pohang, Korea, 2002), p. 40.

[2] Y. J. Chen, G. J. Caporaso, and A. C. Paul, in Proceedings of the 19th International Linear Accelerators Conference, Chicago, IL, 1998 (NTIS, Springfield, VA, 1998), p. 472.

[3] C. Vermare, H. A. Davis, and D. C. Moir, Phys. Plasmas 10, 277 (2003).

[4] J. Zhu et al., IEEE Trans. Plasma Sci. 38, 2873 (2010).

[5] M. J. Burns et al., in Proceedings of the 18th Particle Accelerator Conference, New York, 1999 (IEEE, New York, 1999), p. 617.

[6] S. Sampayan et al., in Proceedings of the Particle Accelerator Conference, Chicago, IL, 2001 (IEEE, New York, 2001), p. 330.

[7] Stanley Humphries, Jr., Charged Particle Beams (Atomic Energy Press, Beijing, 1999).

[8] Y. T. Xie, L. W. Zhang, and J. Zhu, High Power Laser Part. Beams 17, 1913 (2005).

[9] X. G. Jiang et al., High Energy Phys. Nucl. Phys. 29, 1095 (2005).

[10] H. C. Miller, IEEE Trans. Electr. Insul. 24, 765 (1989). 